\title{
Evaluación del consumo de antimicrobianos en 15 hospitales chilenos. Resultados de un trabajo colaborativo, 2013
}

\author{
Isabel Domínguez, Ruth Rosales, Ángela Cabello, Luis Bavestrello y Jaime Labarca \\ en representación del Grupo Colaborativo de Resistencia Bacteriana, Comité de Antimicrobianos \\ de la Sociedad Chilena de Infectología y Centros colaboradores
}

\section{Evaluation of antimicrobial consumption en 15 Chilean hospitals. Results of a collaborative work, 2013}

Surveillance of antimicrobial consumption is a central part in programs of antibiotic stewardship. However, in Chile there are no national data on antibiotic consumption representing a significant number of hospitals by clinical services. In 2013 a survey was sent to multiple Chilean hospitals to evaluate antimicrobial consumption in medical services (MS), surgery services (SS) and critical care units (ICU). We used the standardized methodology recommended by the WHO, using the number of DDD/100 days beds. In the MS and SS beta-lactam and no beta-lactam antibiotics commonly used were evaluated. In the ICU consumption vancomycin, linezolid, imipenem, meropenem, colistin and tigecycline was evaluated. Fifteen hospitals reported the density of antimicrobial consumption. Ceftriaxone and cloxacillin were the most commonly used antibiotics in general services (average cloxacillin 4,9 $\mathrm{DDD} / 100$ bed days in MS and 8,0 DDD/100 in SS; ceftriaxone 13,5 DDD/100 in MS and 16,7 DDD/100 in SS). In the SS there was also a significant consumption of metronidazole (average 14,5 DDD/100 bed days). In the ICU there was an important variability of consumption of selected antibiotics. This study reports the average and range of antibiotic consumption in MS, SS, and ICU from a significant number of hospitals in the country, during 2013. This information allows hospitals to compare their consumption of antibiotics with a significant sample of Chilean hospitals. Analysis of this information should consider a careful interpretation according to the sample shown here and the reality of each hospital.

Key words: Antimicrobial consumption, antimicrobial stewardship, defined daily doses, antimicrobial resistance.

Palabras clave: Consumo de antimicrobianos, control de antimicrobianos, dosis diaria definida, resistencia antimicrobiana.

\section{Introducción}

$\mathrm{E}$ 1 desarrollo de antimicrobianos ha sido uno de los grandes hitos de la medicina moderna, permitiendo una significativa reducción de la morbi-mortalidad de diferentes infecciones. Sin embargo, su uso no siempre es el apropiado. Está demostrado que los antibacterianos se usan de manera innecesaria en animales y también en humanos, lo que se traduce en mayores costos, riesgo de efectos secundarios indeseados y la aparición de resistencia microbiana. Este incremento de la resistencia se asocia a su vez con un mayor consumo de antimicrobianos, en especial de aquellos para el tratamiento de bacterias multiresistentes, creando así un círculo vicioso. El empleo indiscriminado e innecesario de los antibacterianos se ha traducido en un aumento progresivo de la resistencia a antimicrobianos, transformándose en un problema de salud pública en el todo el mundo. Chile no está ajeno a esta realidad; datos de susceptibilidad antimicrobiana de distintos hospitales del país correspondientes al año 2012 muestran una disminución de la susceptibilidad in vitro en cocáceas grampositivas y bacilos gramnegativos (BGN) en comparación con el reporte del año 2009, a excepción de Pseudomonas aeruginosa, que permanece con susceptibilidad in vitro estable $\mathrm{e}^{1,2}$.

Este hecho, sumado a la falta de desarrollo e investigación de nuevos antibacterianos, hacen predecir un futuro sombrío para el tratamiento de las infecciones por bacterias multi-resistentes, caracterizado por una disminución progresiva de las alternativas de tratamiento antimicrobiano y el "re-descubrimiento" de antimicrobianos cuyo uso se había prácticamente abandonado por múltiples motivos, incluyendo la toxicidad. Por tanto, es imprescindible la monitorización del consumo de los antimicrobianos, tanto en la comunidad como en el ámbito hospitalario, sumado a una educación continua acerca del uso adecuado de estos fármacos. En Chile, los últimos datos de consumo de antibacterianos parenterales son del
Clínica Santa María. Santiago Unidad de Infectología (ID) Hospital Barros Luco Trudeau. Santiago. Unidad de Paciente Crítico (RR).

Hospital Gustavo Fricke. Viña del Mar. Unidad de Farmacia (AC). Clínica Reñaca. Viña del Mar (LB) Pontificia Universidad Católica de Chile. Escuela de Medicina, Departamento de Enfermedades Infecciosas del Adulto (JL).

Los autores declaran no tener conflictos de interés en relación a la confección de este manuscrito.

Recibido: 26 de abril de 2016

Correspondencia a:

Jaime A. Labarca

jlabarca@med.puc.cl

Centros colaboradores (de norte a sur del país):

Hospital Dipreca. Santiago.

Q.F. Mónica Alfaro.

Hospital del Salvador. Santiago.

Q.F. Cristián Paredes y Dr. Jaime

Villarroel.

Hospital San Juan de Dios.

Santiago. Dr. José M. Arancibia.

Hospital Padre Hurtado. Santiago.

Dra. Mabel Aylwin.

Clínica Las Condes. Santiago.

Dr. Rodrigo Blamey.

Hospital Barros Luco Trudeau.

Santiago. Q.F. Claudia Benavides y

Q.F. Fernando López.

Hospital Dr. Gustavo Fricke.

Viña del Mar. Q.F. Valeria Gómez

Hospital Carlos Van Buren.

Valparaíso. Dr. Gonzalo Wilson.

Q.F. Rosa Salazar.

Hospital Quilpue. Q.F. María

Santibáñez.

Hospital Guillermo Grant

Benavente. Concepción. Dra. Gisela

Riedel y Q.F. Priscila Campos.

Hospital Higueras. Talcahuano.

Dr. Álvaro Llancaqueo y Q.F. Pamela Toloza.

Hospital Hernán Henríquez Aravena. Temuco. Dra Carolina Chaín y Q.F. Yasna Betancur. Hospital de Valdivia. Dr. Mario Calvo y Q.F. Daniel Muñoz.

Hospital Osorno. Q.F. Soraya Daza. Hospital de Punta Arenas. Q.F.

Renato Carrasco. 
año $2005^{3}$, mientras que los últimos datos de consumo de antibacterianos en la comunidad fueron publicados el año 2011, e incluían el análisis del período entre los años 2000-20084. La Sociedad Chilena de Infectología (SOCHINF) cuenta con un Comité de Antimicrobianos, el cual se ha propuesto, entre otras actividades, evaluar el consumo de antimicrobianos, particularmente en las unidades de pacientes críticos. Además, SOCHINF cuenta con el Grupo Colaborativo de Resistencia Bacteriana (GCRB, Chile), una entidad que agrupa a distintos especialistas del ámbito de la Infectología, Microbiología y Química-Farmacia, y que se reúne periódicamente durante el año y tiene una reunión anual con todos los participantes a fin de analizar los datos epidemiológicos locales, y revisar temas de actualización en resistencia antimicrobiana. Este grupo además recolecta datos de consumo de antimicrobianos y susceptibilidad a los distintos antibacterianos a través de dos encuestas, que se envían a 32 centros hospitalarios del país, y presenta estos datos analizados a los asistentes. Esta red incluye hospitales y servicios pediátricos; sin embargo, en esta evaluación sólo se incluyen servicios de hospitalización de adultos, debido a que no hay acuerdo en la metodología para la evaluación del uso de antimicrobianos en pacientes pediátricos.

El objetivo de este artículo es presentar información del consumo de antimicrobianos de distintos centros hospitalarios de Chile durante el año 2013, recolectados tanto por el Comité de Antimicrobianos como por el GCRB.

\section{Métodos}

Durante el año 2014, SOCHINF realizó a través del Comité de Antimicrobianos y del GCRB, la consolidación de los datos de consumo de antimicrobianos de distintos hospitales del país. La solicitud de información del consumo de antimicrobianos del año 2013 se efectuó utilizando una plantilla prediseñada que establece para los antimicrobianos en consulta las formas farmacéuticas de presentación, la cantidad expresada en gramos para cada forma farmacéutica, el número de camas del servicio clínico y su índice ocupacional en el período. Se solicitó la información a un total de 32 hospitales; se recibió información de 15 hospitales, quienes aportaron datos representativos de los servicios clínicos de Medicina, Cirugía y de Unidad de Paciente Crítico (UPC).

La cantidad de Dosis Diaria Definida (DDD) por 100 días cama es un indicador ampliamente recomendado por la Organización Mundial de la Salud (OMS) como instrumento para analizar de manera comparativa la utilización de medicamentos. Su cálculo requiere de la utilización de la DDD específica para cada antimicrobiano, y que corresponde a una dosis convencional que pretende reflejar la dosis diaria de mantenimiento utilizada en su indicación principal en un paciente adulto y sin considerar los ajustes individuales que pueden ser requeridos en casos particulares. Se encuentra estandarizada por el Centro Colaborador de la OMS en Metodología Estadística de los Medicamentos de Oslo, Noruega ${ }^{5}$. La DDD específica se expresa en gramos, miligramos o unidades internacionales diarias, dependiendo de la presentación de la forma farmacéutica de cada antimicrobiano.

El cálculo del consumo de antimicrobiano se realiza sumando el total unidades (p. ej.: gramos, millones) utilizados de un antimicrobiano en un período de tiempo, dividido por su DDD específica expresada en igual unidad y ajustado por el número de camas ocupadas. Su resultado se expresa como el número de DDD por 100 días cama, estimación interpretada como el número de pacientes tratados diariamente con un determinado antibacteriano por cada 100 camas ocupadas a la dosis establecida.

Se solicitaron las DDD/100 días-cama de los siguientes antimicrobianos en su formulación parenteral (en estricto orden alfabético): amikacina, ampicilina/sulbactam, cefepime, cefotaxima, ceftazidima, ceftriaxona, ciprofloxacina, clindamicina, cloxacilina, colistín, ertapenem, imipenem, linezolid, meropenem, metronidazol, piperacilina/tazobactam, tigeciclina y vancomicina.

\section{Resultados}

Se recibió información de consumo de antimicobianos de 15 hospitales; sin embargo, no todos ellos aportaron datos de todos los antimicrobianos solicitados. Se presenta la información de DDD por cada 100 días cama, en dos formatos. Para los servicios generales, como Medicina y Cirugía se presentan los datos de múltiples antimicrobianos divididos en dos grupos ( $\beta$-lactámicos y no $\beta$-lactámicos). Para cada antimicrobiano se muestra el promedio y rango de consumo. Para las unidades de paciente crítico se seleccionaron antibacterianos específicos de alta relevancia, ya sea por su uso en el tratamiento de bacterias multi-resistentes, por su alto costo, o porque son antibacterianos de última línea para manejo de infecciones y que es necesario cuidar.

En la Figura 1 se muestra el consumo de antimicrobianos $\beta$-lactámicos en los servicios de Medicina. Ceftriaxona es el antimicrobiano más consumido, seguido de cloxacilina; el consumo de ambos antimicrobianos es bastante dispar entre las distintas instituciones, lo que se ve reflejado en el amplio rango de consumo. Destaca además que el consumo de carbapenémicos es bastante similar entre las distintas instituciones, siendo imipenem el más consumido.

En la Figura 2 se muestra el consumo de antimicrobianos no $\beta$-lactámicos seleccionados (amikacina ciprofloxacina, clindamicina, metronidazol y vanco- 


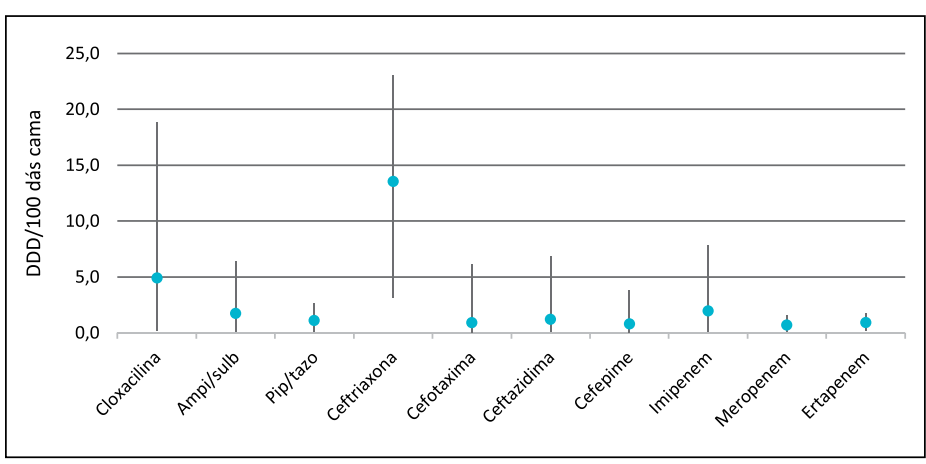

Figura 1. Consumo de antimicrobianos $\beta$-lactámicos en servicios de Medicina en 14 hospitales, expresados en promedio y rango de consumo para cada antimicrobiano, correspondientes al año 2013.

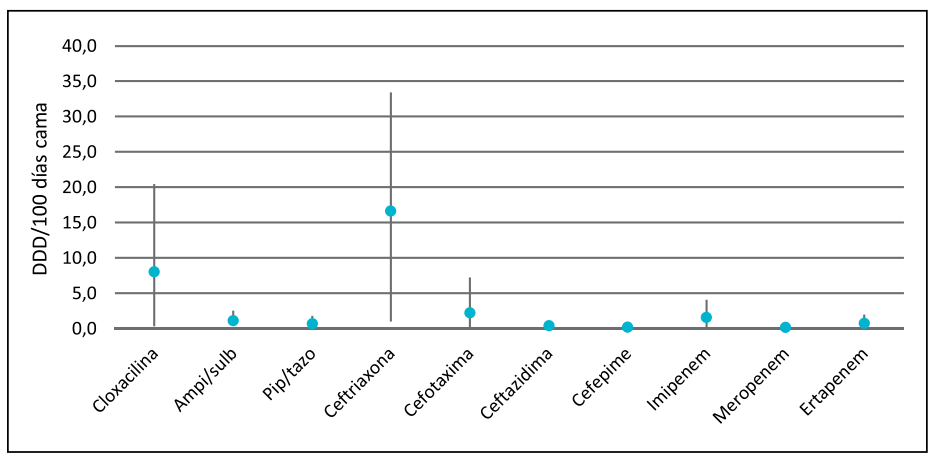

Figura 3. Consumo de antimicrobianos $\beta$-lactámicos en servicios de Cirugía en 12 hospitales, expresados en promedio y rango de consumo para cada antimicrobiano, correspondientes al año 2013.

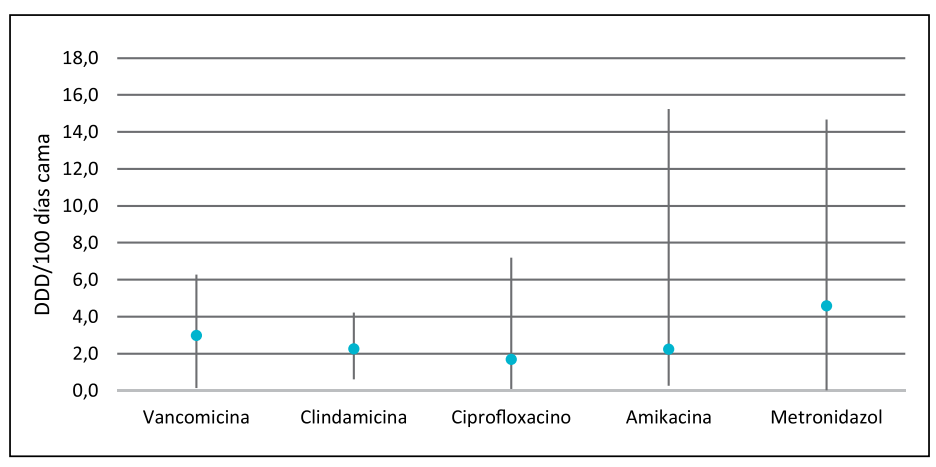

Figura 2. Consumo de antimicrobianos no $\beta$-lactámicos en servicios de Medicina en 14 hospitales, expresados en promedio y rango de consumo para cada antimicrobiano correspondientes al año 2013.

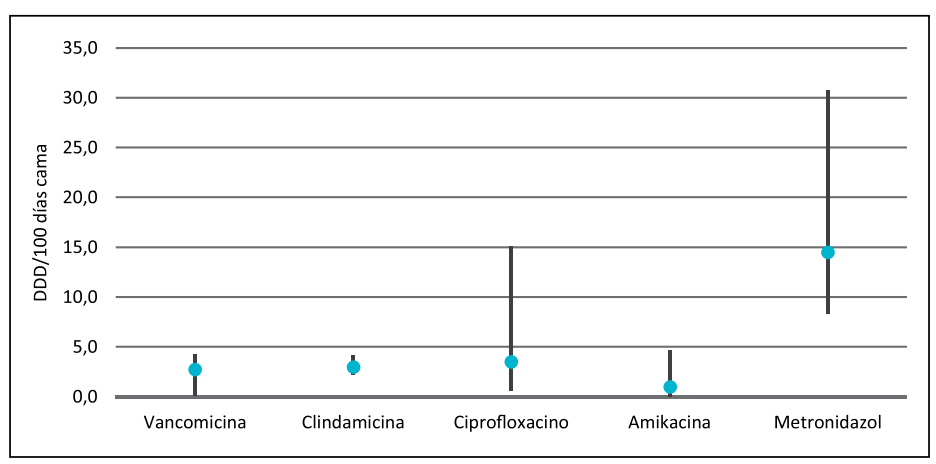

Figura 4. Consumo de antimicrobianos no $\beta$-lactámicos en servicios de Cirugía en 12 hospitales, expresados en promedio y rango de consumo para cada antimicrobiano, correspondientes al año 2013. micina) en los servicios de Medicina. Los promedios de consumo de dichos antimicrobianos son similares; destaca el amplio rango de consumo entre los distintos centros hospitalarios.

En la Figura 3 se muestra el consumo de antimicrobianos $\beta$-lactámicos en los servicios de Cirugía. Al igual que en los servicios de Medicina, ceftriaxona es el antimicrobiano de mayor consumo, siendo éste muy dispar entre las distintas instituciones. A diferencia de los servicios de Medicina, prácticamente no hay consumo de ceftazidima, lo que refleja probablemente las diferencias de las patologías que se manejan en los servicios de Medicina versus los de Cirugía. En la Figura 4 se muestra el consumo de antimicrobianos no $\beta$-lactámicos seleccionados (amikacina ciprofloxacina, clindamicina, metronidazol vancomicina) en los servicios de Cirugía. Destaca el consumo de metronidazol, que corresponde al segundo antimicrobiano de mayor consumo en los servicios de Cirugía (a diferencia de cloxacilina en los servicios de Medicina); también se observa un mayor consumo de ciprofloxacina y un menor consumo de amikacina, en relación a los servicios de Medicina.
En relación a las unidades de paciente crítico, se obtuvo la información de 10 UPC de hospitales de alta complejidad, de los cuales siete corresponden a hospitales regionales y tres de la Región Metropolitana; todos ellos con un número de camas mayor a 350 camas y tres con número mayor a 600 camas. Las Figuras 5 y 6 muestran el consumo de antimicrobianos para cocáceas grampositivas en UPC. El consumo de vancomicina en las distintas unidades de paciente crítico es relativamente uniforme. Sin embargo, en la Figura 6 se aprecia que linezolid tiene un uso muy variado en las distintas UPC, destacando unidades en el que el consumo es muy bajo $y$ otras en que el consumo es elevado. Probablemente reflejan diversas realidades de resistencia bacteriana y criterios de uso.

En la Figura 7 se muestra el consumo de carbapenémicos, destacando que en la mayoría de las UPC se usan imipenem y meropenem, siendo en general mayor el consumo de imipenem, que además se presenta más uniforme. Sin embargo, meropenem tiene un consumo más variable en las distintas UPC. No se observa reemplazo de un carbapenémico por otro. 
En la Figura 8 se observa el consumo de colistín, el que es muy diferente en las distintas UPC, probablemente demostrando realidades epidemiológicas diversas en relación a microorganismos resistentes a carbapenémicos.
En la Figura 9 se observa el consumo de tigeciclina en estas unidades, el que es bajo, en general.

Finalmente, en la Figura 10 se muestra un consolidado del consumo de estos antimicrobianos en UPC, mostrando el promedio y el rango de dispersión de uso.

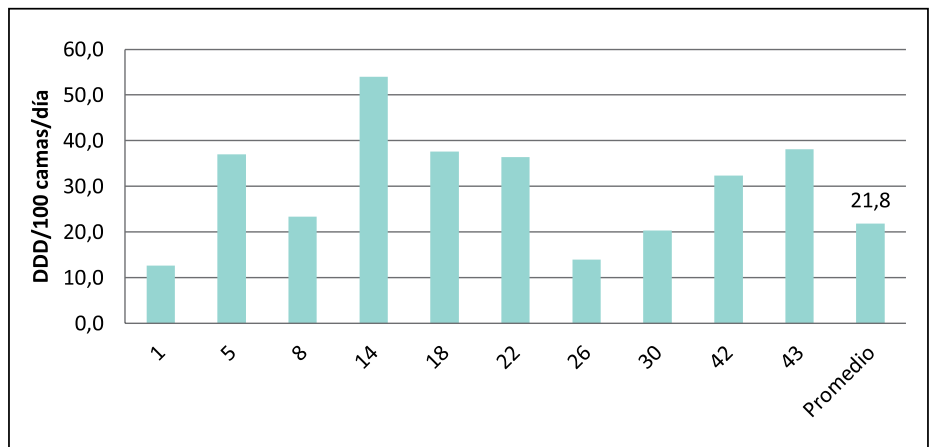

Figura 5. Consumo de vancomicina en UPC en 10 hospitales, expresado para cada hospital y en promedio, correspondiente al año 2013.

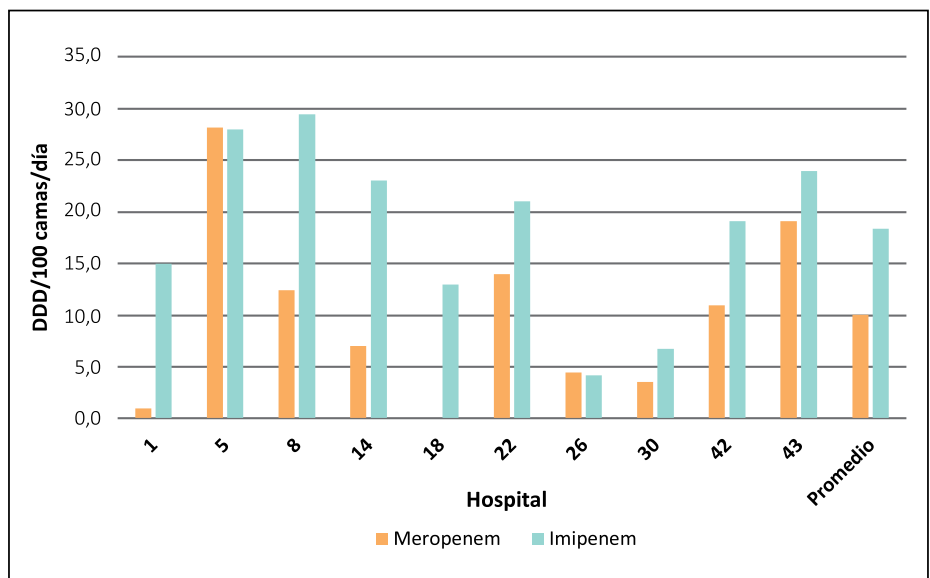

Figura 7. Consumo de imipenem y meropenem en UPC en 10 hospitales, expresado para cada hospital y en promedio, correspondiente al año 2013.

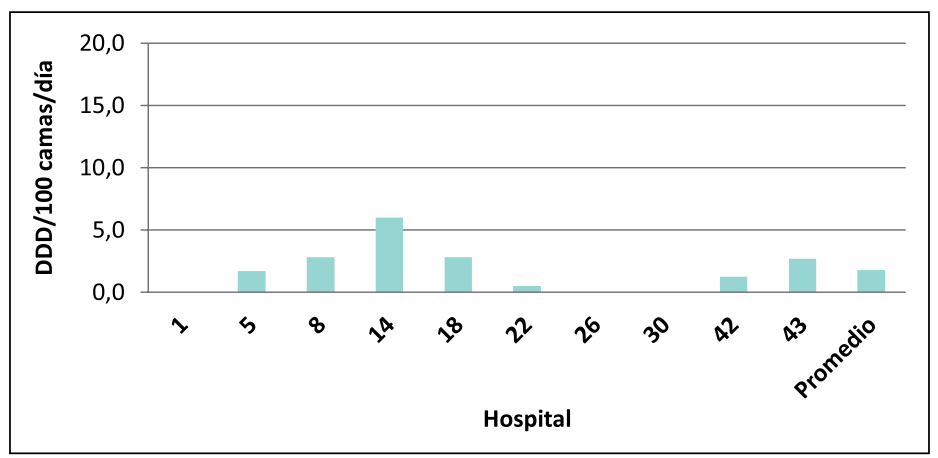

Figura 9. Consumo de tigeciclina en UPC en 10 hospitales, expresado para cada hospital y en promedio, correspondiente al año 2013.

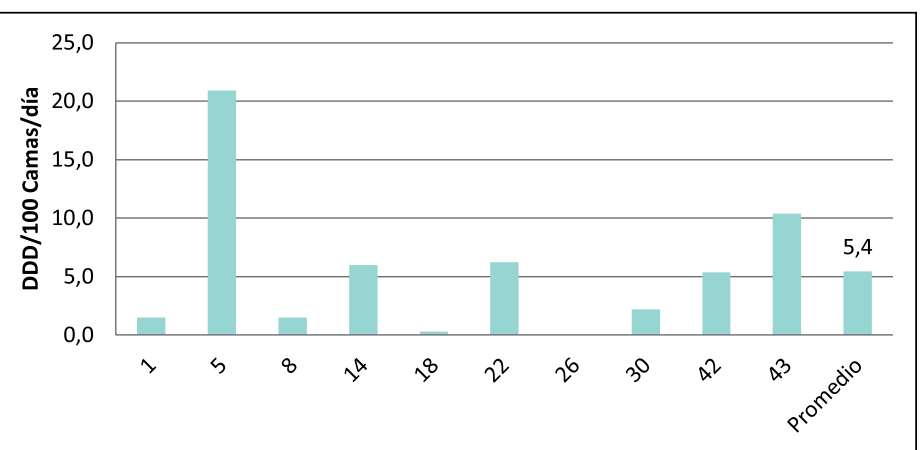

Figura 6. Consumo de linezolid en UPC en 10 hospitales, expresado para cada hospital y en promedio, correspondiente al año 2013.

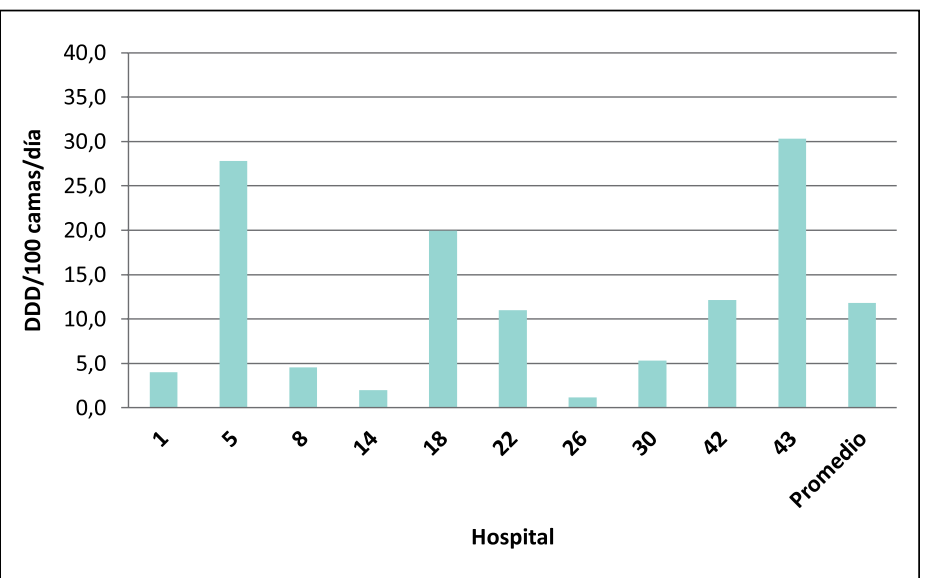

Figura 8. Consumo de colistín en UPC en 10 hospitales, expresado para cada hospita y en promedio, correspondiente al año 2013.

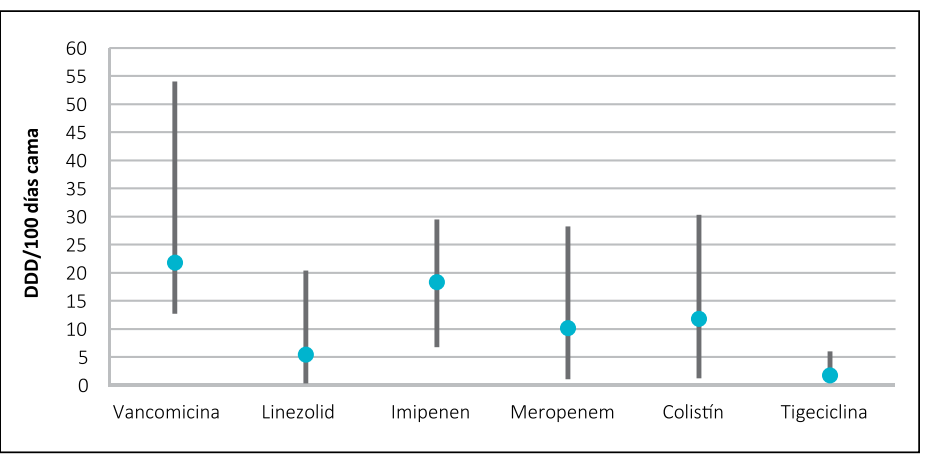

Figura 10. Consumo de antimicrobianos seleccionados en UPC en 10 hospitales, expresados en promedio y rango de consumo para cada antimicrobiano, correspondientes al año 2013. 


\section{Discusión}

La evaluación del consumo de antimicrobianos usando una metodología estandarizada es muy importante para cada hospital. Permite la evaluación del consumo general de antimicrobianos en los hospitales pero, más importante es focalizar el consumo en los distintos servicios clínicos, el que esta comandado tanto por la patologías de los pacientes, la epidemiología microbiológica local y las normas de uso de antimicrobianos de cada unidad. Esto permite caracterizar el consumo e identificar las diferencias de consumo en cada una de las distintas áreas de los hospitales, y hacer un seguimiento en el tiempo. De esta manera, es posible evaluar variaciones en el tiempo, que eventualmente requieran o hayan requerido de alguna intervención.

En este trabajo se presentan los datos de un número significativo de hospitales chilenos, la mayoría de alta complejidad, y que representan la realidad de las distintas regiones del país. Al usar una metodología estandarizada, permite realizar comparaciones para cada antimicrobiano entre los distintos servicios clínicos de los diferentes hospitales. Aquí se presentan los datos para los servicios de Medicina, Cirugía y UPC. En Medicina es especialmente relevante el uso de ceftriaxona y cloxacilina, mientras que en Cirugía, el uso de ceftriaxona y metronidazol. En las UPC se observa un incremento del uso de antibacterianos para tratar bacterias multi-resistentes, aumentando significativamente el uso de vancomicina y carbapenémicos. En relación a los servicios clínicos de UPC aparece el uso de linezolid y colistin, lo que esta en relación al problema de multi-resistencia bacteriana actual: Staphylococcus aureus resistentes a meticilina y resistencia a vancomicina en Enterococcus spp en el caso de linezolid y a carbapenémicos en BGN, en el caso de colistín. A pesar de que existen publicaciones previas en relación al consumo de antibacterianos en hospitales en Chile, la información previamente reportada representa el consumo global de antimicrobianos y no es posible compararla con aquella presentada por este artículo, que se muestra por los distintos servicios clínicos.

La información contenida en las figuras en los que se ve el promedio de los hospitales y el rango de dispersión del consumo para los distintos antimicrobianos permite a los hospitales de todo el país establecer una comparación con la información recolectada en esta muestra, permitiéndoles evaluarse y eventualmente, si están en un rango alto de uso de determinado antimicrobiano, requiere buscar explicaciones de diferencias con el referente nacional disponible en este artículo. Cabe señalar que un determinado consumo de antimicrobiano a nivel institucional, que se encuentre en rango superior, no necesariamente indica un uso inapropiado, sino que refleja una situación que requiere un análisis por cada hospital, en relación a su realidad epidemiológica local. No obstante, es posible que en algunas situaciones pudiesen estar asociadas a un uso inadecuado de antimicrobianos o falta de cumplimiento de las normas de la institución.

Este informe tiene algunas limitaciones que vale la pena considerar. La información recopilada sólo representa un porcentaje de los hospitales a los cuales se les solicitó información. Además, algunos de los hospitales sólo reportaron información de algunos antimicrobianos (y no de todos los solicitados), pudiendo haber un sesgo de información. El universo de los hospitales que reportaron datos de servicios generales no necesariamente corresponde a los mismos hospitales que aportaron datos de UPC, dado que se solicitaron por separado. Hay antibacterianos que no fueron incorporados en este reporte, tal como cefazolina, la que es muy importante por su rol en profilaxis antimicrobiana, levofloxacina y moxifloxacina, que son relevantes en el tratamiento de infecciones respiratorias bajas. Este reporte sólo considera antimicrobianos parenterales y no orales, que son muy importantes en el ámbito comunitario.

En las circunstancias actuales, comandadas por un mundo globalizado, donde la resistencia bacteriana ha cobrado un rol muy significativo y de difícil control, la vigilancia del consumo de antimicrobianos en el hospital es fundamental, tanto para evaluar el cambio epidemiológico que impacta en el consumo antimicrobianos, como también a su vez evaluar las desviaciones del uso que pueden influir en un agravamiento de la resistencia bacteriana actual. Así, estos datos permiten tener una mirada general del consumo de antimicrobianos en pacientes hospitalizados en nuestro país, y si bien da una visión parcial, permite a cada hospital establecer una comparación para algunos servicios clínicos en algunos antibacterianos determinados, conteniendo una información valiosa para estos hospitales. Esta información es además especialmente útil para evaluar impactos en intervenciones realizadas en la misma institución. En el futuro debiéramos ser capaces de reproducir esta información para establecer las necesarias comparaciones temporales y de tendencia en nuestro país.

\section{Resumen}

La vigilancia del consumo de antimicrobianos es parte central en los programas de utilización de antimicrobianos. Sin embargo, en Chile no se conocen datos del consumo de antibacterianos por servicios clínicos, que representen un número importante de hospitales. En el año 2013 se envió una encuesta a múltiples hospitales chilenos para evaluar el consumo de antimicrobianos en los servicios clínicos de Medicina (SM), Cirugía (SC) y Unidades de Pacientes Críticos (UPC). Para ello se usó la metodología estandarizada recomendada por la OMS de DDD/100 
días camas. En los SM y SC se evaluaron antibacterianos $\beta$-lactámicos y no $\beta$-lactámicos de uso frecuente. En las UPC se evaluó el consumo de vancomicina, linezolid, imipenem, meropenem, colistín y tigeciclina. Se obtuvo resultados de 15 hospitales, los cuales informaron la densidad de consumo de antimicrobianos de los servicios mencionados. Ceftriaxona y cloxacilina fueron los antimicrobianos más utilizados en servicios generales (promedio cloxacilina 4,9 DDD/100 días cama en SM y 8,0 DDD/100 en SC; ceftriaxona 13,5 DDD/100 en SM y 16,7 $\mathrm{DDD} / 100$ en SC). En los SC se agrega además un consumo importante de metronidazol (promedio 14,5 DDD/100 días cama). En las UPC destaca la variabilidad de consumo de antimicrobianos seleccionados. Este estudio reporta el promedio y rangos de consumo de antibacterianos en $\mathrm{SM}, \mathrm{SC}$ y UPC de un número importante de hospitales del país durante el año 2013. Esta información permite a los hospitales comparar su consumo de antibacterianos con una muestra significativa de hospitales chilenos. El análisis de esta información debe considerar una cuidadosa interpretación de acuerdo a la muestra aquí representada y la realidad de cada uno de los hospitales.

\section{Referencias bibliográficas}

1.- Cifuentes M, Silva F, García P, Bello H, Briceño I, Calvo-A M y cols. Susceptibilidad antimicrobiana en Chile 2012. Rev Chilena Infectol 2014; 31 (2): 123-30.

2.- Silva F, Cifuentes M, Pinto M E. Resultados de la vigilancia de susceptibilidad antimicrobiana en Chile: Consolidando una red. Rev Chilena de Infectol 2011; 28 (1): 19-27.

3.- Fica A, Cabello A, Juliet C, Prado P, Bavestrello L. Consumo de antibióticos parenterales en diferentes hospitales de Chile durante el año 2005. Rev Chilena Infectol 2008; 25 (6): 419-27.

4.- Bavestrello L, Cabello A. Consumo comunitario de antimicrobianos en Chile, 2000-2008. Rev Chilena Infectol 2011; 28 (2): 107-12.

5.- WHO Collaborating Centre for Drug Statistics Methodology, Guidelines for ATC classification and DDD assignment 2012. Oslo 2011.

Disponible en: http://www.who.no/atc_ddd publications/guidelines/. (Accedido: 25 de abril de 2016). 